\title{
TOTALLY DISCONNECTED COMPACTIFICATIONS
}

\section{PARFENY P. SAWOROTNOW}

Department of Mathematics

The Catholic University of America

Washington, DC 20064

United States of America

(Received September 28, 1992 and in revised form June 23, 1993)

\begin{abstract}
There is a one-to-one order preserving correspondence between totally disconnected compactifications of a topological space and certain Boolean algebras of open closed subsets on it.
\end{abstract}

KEY WORDS AND PHRASES. Gelfand theory, Stone spaces, Boolean rings, Boolean algebras. totally disconnected spaces, totally disconnected compactifications, Hausdorff compactifications, Stone Representation Theorem.

1991 AMS SUBJECT CLASSIFICATION CODES. Primary 54D35; Secondary 06E15.

\section{INTRODUCTION.}

One of the interesting consequences of Gelfand theory is the correspondence between compactifications of a completely regular space $S$ and certain subalgebras of the algebra $C(S)$ of all bounded continuous complex-valued functions on it. This correspondence is described in $\S 43$ of Gel'fand et al. [1]. Theorem 1 in $§ 43$ of Gel'fand et al. [1] states that each Hausdorff compartification of $S$ corresponds to a subalgebra $A$ of $C(S)$ which is closed under involution and is regular in the sense that members of $A$ separate points from closed subsets. .

In this paper we shall show that there is a similar consequence of Stone theory about Boolean rings. We shall prove that there is one-to-one order preserving correspondence between certain compactifications of a totally disconnected space $S$ and certain Boolean rings of open-closed subsets of the space $S$. (In fact, we would be justified in referring to those Boolean rings as regular Boolean rings (members of these rings also separate points and closed sets)). There is an interesting analogy between consequences of Gelfand and Stone's theories.

2. MAIN RESULT.

Let $S$ be a totally disconnected space (see Simmons [6, p 149]). This means that for any $s, t \in S$ there is an open closed subset of $S$ containing $s$ but excluding $t$ (Note that $S$ is a Hausdorff space). (Note also that our definition differs somewhat from definitions given in Johnstone [2], Koppelberg [4] and Monk and Bonnet [5].) Let $\mathcal{L}(S)$ be the space of all open closed subsets of $S$. Then $\mathcal{L}(S)$ is a Boolean algebra (Simmons [6, p 345]) under standard set theoretic operations (as well as a Boolean ring under certain algebraic operations (symmetric differences and intersections)).

The main result deals with those compactifications of $S$ that are totally disconnected. Ac- 
cording to Kelley [3, p 151] a compactification of $S$ is a compact space $Q \supset S$ such that $S$ is dense in $Q$ and the topology of $S$ is the (relative) topology induced by $Q$.

THEOREM 1. For each totally disconnected compactification $Q$ of $S$ there exists a Boolean subring $L(Q)$ of $\mathcal{L}(S)$ with the following properties:

(1) $L(Q)$ is isomorphic to the Boolean ring $\mathcal{L}(Q)$ of all open closed subsets of $Q$.

(2) For each open subset $O$ of $S$ and $s \in O$ there exists $O^{\prime} \in L(Q)$ such that $s \in O^{\prime} \subset O$.

Conversely, for each Boolean subring $L$ of $\mathcal{L}(S)$ with property (2) there exists a totally disconnected compactification $Q(L)$ such that $L$ is isomorphic to $\mathcal{L}(Q(L))$.

PROOF. Let $Q$ be a totally disconnected compactification of $S$. Consider the family

$$
\begin{gathered}
L(Q)=\{G \cap S: G \text { is an open-closed subset of } Q\}, \\
L(Q)=\{G \cap S: G \in \mathcal{L}(Q)\} .
\end{gathered}
$$

Then $L(Q)$ is a Boolean algebra isomorphic to $\mathcal{L}(Q)$ (under the correspondence $G \cap S \leftrightarrow G$ ) (the fact that the correspondence " $G \cap S \leftrightarrow G$ " is one-to-one follows from the fact that each $G \in \mathcal{L}(Q)$ is both open and closed, and $S$ is dense). Using Theorem $\mathrm{C}$ on page 150 of Simmons [6], it is easy to verify that $L(Q)$ satisfies property (2).

Now let $L$ be any Boolean subalgebra of $\mathcal{L}(S)$ satisfying (2). Let $Q(L)$ be the set of al] non-zero homomorphisms $q$ of $L$ into $Z_{2}=\{0,1\}$ (the smallest Boolean ring (which is also a field)). Then $Q$ is totally disconnected compact space with respect to the topology whose base $\beta$ can be described as follows (Simmons [6, p 351]): for each non-empty $G \in L$ consider the set

$$
U_{G}=\{q \in Q: q(G)=1\}
$$

and then take $\beta$ to be the family of all sets of the form $U_{G}$,

$$
\beta=\left\{U_{G}: G \in L, G \neq \phi\right\}
$$

One can identify each $s \in S$ with the member $q_{s}$ of $Q$ defined by

$$
q_{s}(G)=\varphi_{G}(s), G \in L
$$

(where $\varphi_{G}$ is the characteristic function of $G: \varphi_{G}(s)=1$ if $s \in G$ and $\varphi_{G}(s)=0$ if $s \notin G$ ). Now note that the map $\psi: s \rightarrow q_{s}$ is one-to-one (because of property (2)) and continuous (since $\psi^{-1}\left(U_{G}\right)=G$ ). Property (2) implies that $\psi$ maps open sets into open sets (every open set in $S$ is a union of members $G$ of $L$ and $\psi(G)=U_{G} \cap \psi(S)$ ).

To show that $S$ (or rather $\psi(S)$ ) is dense in $Q$, consider any $q_{0} \in Q$ and any open subset $O$ of $Q$ with $q_{0} \in Q$. Take $G \in L, G \neq \phi$, such that $q_{0} \in U_{G} \subset O$. Then $U_{G}$ contains plenty of members of the form $q_{s}$ with $s \in S$. This means that $S$ is dense in $Q$.

Thus $Q$ is a compactification of $S$. It is not difficult to show that $L$ is isomorphic to $\mathcal{L}(Q)$ (in fact, this is the consequence of Stone Representation Theorem on p. 351 in Simmons [6]).

Now let $\mathcal{F}$ be the class of all totally disconnected compactifications of $S$ and let $\Sigma$ be the class of all Boolean subalgebras of $\mathcal{L}(S)$ satisfying condition (2) of Theorem 1 above. Define the partial order " $\leq$ " on $\mathcal{F}$ as it is done on page 151 of Kelley [3]: if $Q_{1}, Q_{2} \in \mathcal{F}$ then $Q_{1} \leq Q_{2}$ if there exists a continuous mapping $T: Q_{2} \rightarrow Q_{1}$ such that $T_{s}=s$ for all $s \in S$. Order $\Sigma$ by inclusion: if $L_{1}, L_{2} \in \mathcal{L}(S)$, then $L_{1} \leq L_{2}$ if $L_{1}$ is a Boolean subalgebra of $L_{2}$.

THEOREM 2. The mappings $Q \rightarrow L(Q)$ and $L \rightarrow Q(L)$ considered in Theorem 1 above are one-to-one and preserve partial orders of $\mathcal{F}$ and $\Sigma$. 
PROOF. First assertion follows from the facts that $Q$ is homeomorphic to $Q(L(Q))$ (follows from Theorem 2, p 380 of Stone [7]) and that $L$ is isomorphic to $L(Q(L))$. (Stone Representation Theorem on page 351 of Simmons [6]).

Let us show that the correspondence $L \leftrightarrow Q(L)$ preserves the partial order. Let $T: Q_{2} \rightarrow Q_{1}$ be a continuous map of a compactification $Q_{2}$ into a compactification $Q_{1}\left(Q_{1}, Q_{2} \in \mathcal{F}, Q_{1} \leq Q_{2}\right)$ such that $T_{s}=s$ for all $s \in S$ (for simplicity we identify members $s$ of $S$ with their corresponding members $q_{s}$ in $Q_{1}$ and $Q_{2}$ ). For $i=1,2$ let

$$
L_{\imath}=\left\{G \cap S: G \in \mathcal{L}\left(Q_{\mathbf{\imath}}\right)\right\}
$$

If $O \in L$, then $O=G \cap S$ for some $G \in \mathcal{L}\left(Q_{1}\right)$. Let

$$
O^{\prime}=T^{-1}(O)=T^{-1}(G \cap S)=T^{-1}(G) \cap S
$$

Then $O^{\prime}$ is both open and closed hence belongs to $L_{2}$. But members of $S$ are invariant under $T$ and the only member of $S$ that maps into a fixed $s \in S$ is itself. This simply means that $O^{\prime}=O$. This proves that $L_{1} \subset L_{2}$ i.e. $L_{1} \leq L_{2}$.

Now assume that $L_{1} \subset L_{2}$ where $L_{1}=\left\{G \cap S: G \in \mathcal{L}\left(Q_{\imath}\right)\right\}, i=1,2$, where $Q_{1}, Q_{2}$ are as above. Then each $q \in Q_{2}$ can be considered as a homomorphism of $L_{2}$ into $Z_{2}=\{0,1\}$. Consider its restriction $T_{q}$ to $L_{1}$. Clearly $T_{q} \in Q_{1}$. Let us show that the map $T: Q_{2} \rightarrow Q_{1}$ is continuous. Let $q_{0} \in Q_{2}$ and let $G \in L_{1}$ be such that

$$
T_{q_{0}} \in U_{G}=\left\{q \in Q_{1}: q(G)=1\right\}
$$

Then $T_{q_{0}}(G)=1$ and $G \in L_{2}$ also. Consider

$$
V_{G}=\left\{q \in Q_{2}: q(G)=1\right\}
$$

Then $T\left(V_{G}\right) \subset U_{G}$ and so $T$ is continuous on $q_{0}$. It is also easy to see that $T_{s}=s$ for each $s \in S$. 3. AN EXAMPLE.

It is clear that total disconnectedness of the space $S$ above does not by itself guarantee existence of Boolean algebras of open closed subsets of $S$ that satisfy condition (2) (in Theorem 1) above. Because of this we give an example of a space which has plenty of Boolean algebras with property (2).

In this paper the term "open interval" means any set of the form

$$
\begin{gathered}
(a, b)=\{x: a<x<b\} \\
(c, \infty)=\{x: c<x\} \\
(-\infty, d)=\{x: x<d\}
\end{gathered}
$$

End points (of an interval) are numbers $a, b, c, d$ (quantities $\infty$ and $-\infty$ are excluded from our considerations).

EXAMPLE. Let $S$ be the set of all transcendental (real) numbers (those real numbers which are not roots of polynomials with integral coefficiants). Then $S$ is totally disconnected with respect to the topology induced by the standard topology of real numbers (the topology, generated by open intervals).

Now let $L_{1}$ be the family of these subsets of $S$ which are finite unions of mutually disjoint open intervals with rational end-points and let $L_{2}$ be the family of those subsets of $S$ which are 
finite unions of disjoint open intervals whose end-points are algebraic numbers. It is easy to see that both $L_{1}$ and $L_{2}$ are Boolean algebras of open closed sets, which satisfy condition (2) of Theorem 1 above. Note also that $L_{1} \subset L_{2}$.

The reader should have no difficulties in constructing other examples. For instance, in the above example, one could replace transcendental numbers by irrationals, the algebraic numbers by rationals and rationals by those of the form $p 2^{-n}$ ( $p$ and $n$ are integers, $n \geq 0$ ).

4. REMARK.

The author feels that it is appropriate to make the following remark concerning completely regular spaces mentioned at the beginning of the paper. As it was already stated above, there is a one-to-one correspondence $Q \leftrightarrow B$ between Hausdorff compactifications $Q$ of a completely regular space $S$ and Banach subalgebras $B$ of $C(S)$ which are closed under conjugation and which separate points and closed sets in $S$. It turns out that this correspondence preserves also the partial order. In other words, if $Q_{1}$ and $Q_{2}$ are two Hausdorff compactifications of $S$ such that $Q_{1} \leq Q_{2}$ (as defined above) and $B_{1}, B_{2}$ are corresponding regular Banach algebras, then $B_{1} \subseteq B_{2}$. Proof of this fact is a straightforward modification of the proof of Theorem 2 above.

\section{REFERENCES}

1. GEL'FAND, I.M., RAĬKOV, D.A. and ŠILOV, G.E., Commutative normed rings, Gosudarstv. Izdat. Fiz.-Mat. Lit., Moscow, 1960, MR 23, A1242.

2. JOHNSTONE, P., Stone spaces, Cambridge Studies in Advanced Mathematics, 3 (Cambridge University Press), MR 85f, 54002.

3. KELLEY, J.L., General Topology, Van Nostrand, 1961.

4. KOPPELBERG, S., Handbook of Boolean Algebras, Vol. 1, North-Holland, 1989, MR 90k, 06002 .

5. MONK, J.D. and BONNET, R., Handbook of Boolean Algebras, Vol. 3, North-Holland, 1989, MR 90k, 06004.

6. SIMMONS, G.F., Introduction to Topology and Modern Analysis, McGraw-Hill, 1963, MR 26, 4145.

7. STONE, M.H., Application of the theory of Boolean rings to general topology, Trans. Amer. Math. Soc. 41 (1937), 375-481. 


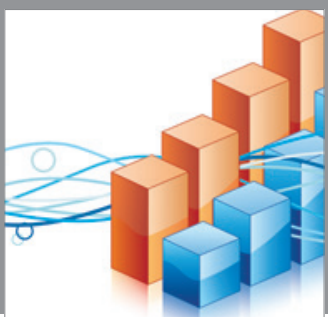

Advances in

Operations Research

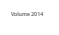

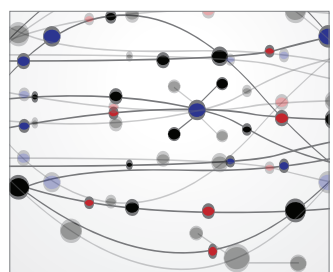

\section{The Scientific} World Journal
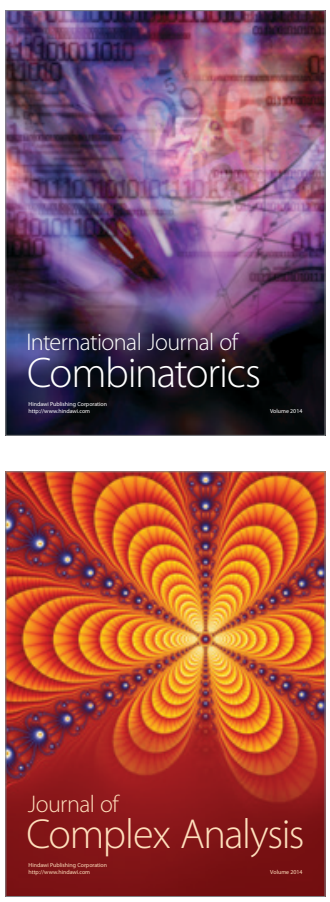

International Journal of

Mathematics and

Mathematical

Sciences
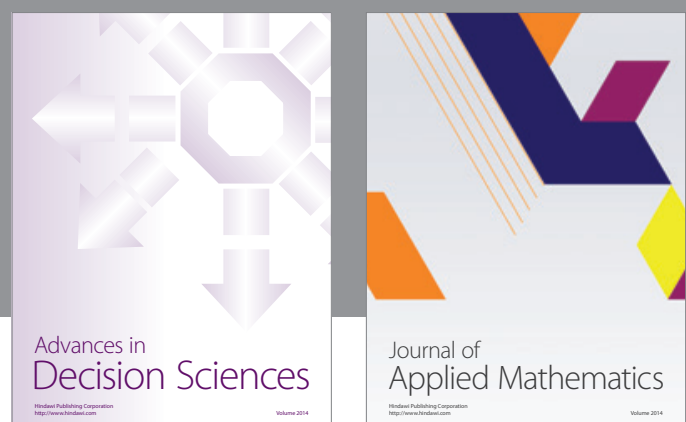

Journal of

Applied Mathematics
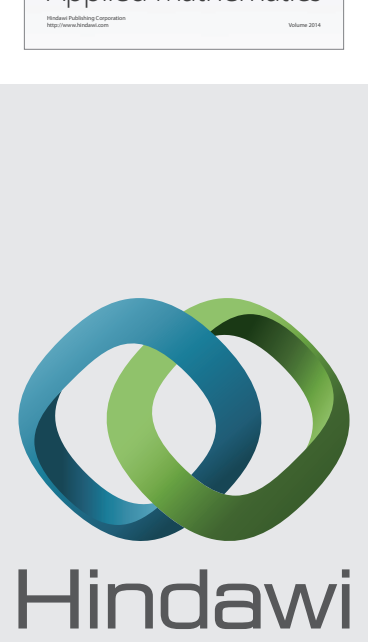

Submit your manuscripts at http://www.hindawi.com
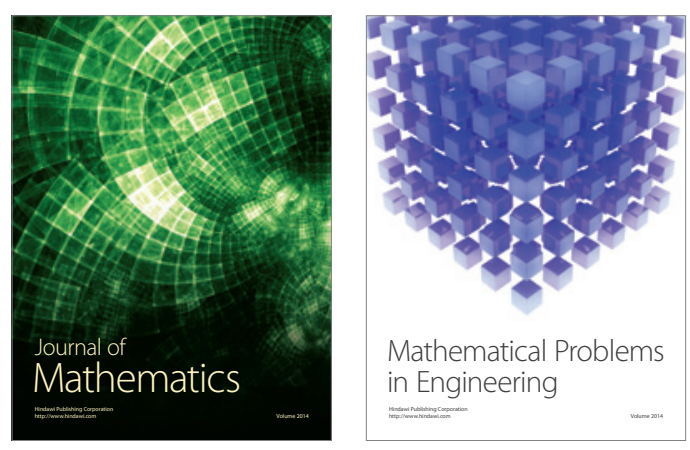

Mathematical Problems in Engineering
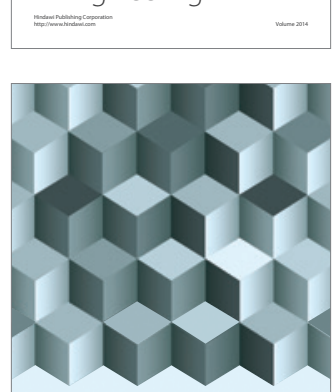

Journal of

Function Spaces
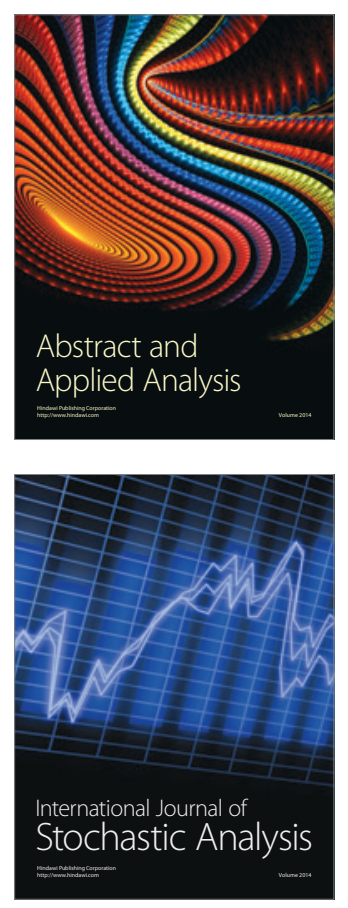

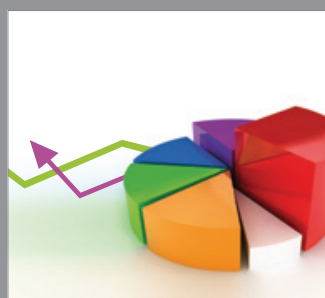

ournal of

Probability and Statistics

Promensencen
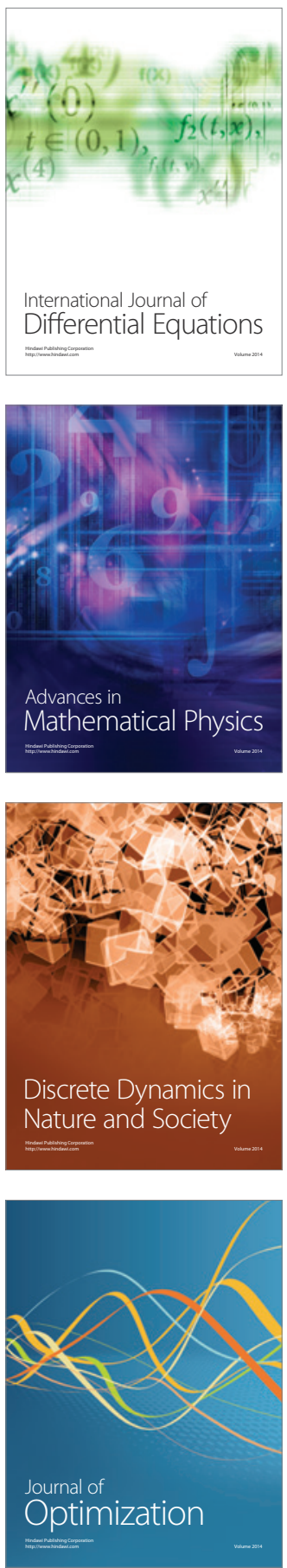